\title{
Tempo de espera ao acesso ambulatorial especializado no pré-natal de alto risco: estudo de método misto
}

\author{
Waiting time for specialized outpatient access in high-risk prenatal care: a mixed-method study \\ Tiempo de espera para el acceso ambulatorio especializado en atención prenatal de alto riesgo: un \\ estudio de métodos mixtos
}

Fabiana Fontana Medeiros

ORCID: https://orcid.org/0000-0002-7876-572X Universidade Estadual de Londrina, Brasil

E-mail: fontana.fabi@hotmail.com

Izabel Dayana de Lemos Santos

ORCID: https://orcid.org/0000-0003-3559-5350

Universidade Estadual de Londrina, Brasil

E-mail: izabellemos87@hotmail.com

Juliana Vicente de Oliveira Franchi

ORCID: https://orcid.org/0000-0001-9441-7736

Universidade Estadual de Londrina, Brasil E-mail: jolliveira@yahoo.com.br

Sebastião Caldeira

ORCID: https://orcid.org/0000-0003-2827-1833 Universidade Estadual do Oeste do Paraná, Brasil E-mail: calenf3@gmail.com

Rosângela Aparecida Pimenta Ferrari ORCID: https://orcid.org/0000-0003-0157-7461 Universidade Estadual de Londrina, Brasil E-mail: ropimentaferrari@uel.br

Alexandrina Aparecida Maciel Cardelli ORCID: https://orcid.org/0000-0002-0222-8821

Universidade Estadual de Londrina, Brasil E-mail: macielalexandrina@gmail.com

\begin{abstract}
Resumo
Objetivo: analisar o tempo de espera ao acesso ambulatorial especializado no pré-natal de alto risco. Metodologia: estudo de método misto com desenho explanatório sequencial. Na etapa quantitativa realizou-se um estudo transversal analítico com 319 puérperas durante sua internação hospitalar, utilizando instrumento semiestruturado e entrevista, seguido de análise descritiva e analítica por meio do Teste Qui-quadrado $(p \leq 0,05)$. Na fase qualitativa o estudo direcionou-se pela Fenomenologia Social com 13 gestores da assistência indireta no pré-natal de alto risco, por meio de entrevista audiogravada face a face e analisados à luz da Fenomenologia Social. Resultados: o período aguardado para acesso ao serviço especializado foi de 15 dias (32,3\%), 16 a 30 dias (27,9\%), acima de 90 dias $(9,4 \%)$ e $(19,1 \%)$ não tiveram acesso ao serviço ambulatorial. Os resultados demonstraram como fatores que influenciam no tempo de espera: insuficiência de vagas, incompletude ou não monitoramento das informações e não atendimento aos critérios de encaminhamento. Os gestores apontaram que a equipe de saúde precisa ter bagagem de conhecimento suficiente para desenvolver suas ações com reciprocidade de intenções no processo cuidar e do cuidado. Conclusão: a primeira consulta em serviço especializado ocorre em tempo prolongado e algumas mulheres não conseguem nem mesmo o acesso ao serviço, resultando no cuidado exclusivo da atenção primária, a qual não está organizada para assumir o cuidado exclusivo à gestante de alto risco.

Palavras-chave: Acesso aos serviços de saúde; Encaminhamento e consulta; Assistência pré-natal, Gestação de alto risco; Gestão de serviços de saúde; Assistência integral à saúde.

Abstract

Objective: to analyze the waiting time for specialized outpatient access for high-risk prenatal care. Methodology: mixed method study with sequential explanatory design. In the quantitative stage, an analytical cross-sectional study was carried out with 319 postpartum women during their hospital stay, using a semi-structured instrument and interview, followed by descriptive and analytical analysis using the Chi-square test $(p \leq 0,05)$. In the qualitative phase, the study was guided by Social Phenomenology with 13 managers of indirect assistance in high-risk prenatal care, through an audio-recorded face-to-face interview and analyzed in the light of Social Phenomenology. Results: the
\end{abstract}


waiting period for accessing the specialized service was 15 days (32,3\%), 16 to 30 days (27,9\%), over 90 days (9,4\%) and $(19,1 \%)$ not had access to the outpatient service. The results showed as factors that influence the waiting time: insufficient vacancies, incomplete or non-monitoring of information and non-compliance with the referral criteria. The managers pointed out that the health team needs to have enough knowledge to develop their actions with reciprocal intentions in the care and care process. Conclusion: the first consultation in a specialized service takes place over a long period of time and some women cannot even access the service, resulting in exclusive care in primary care, which is not organized to assume exclusive care for high-risk pregnant women.

Keywords: Health services accessibility; Referral and consultation; Prenatal care; Pregnancy, high-risk; Health services administration; Comprehensive health care.

\section{Resumen}

Objetivo: analizar el tiempo de espera para el acceso ambulatorio especializado para atención prenatal de alto riesgo. Método: estudio de método mixto con diseño explicativo secuencial. En la etapa cuantitativa, se realizó un estudio analítico transversal con 319 puérperas durante su estadía hospitalaria, mediante instrumento semiestructurado y entrevista, seguido de análisis descriptivo y analítico mediante la prueba de Chi-cuadrado $(p \leq 0,05)$. En la fase cualitativa, el estudio fue guiado por Fenomenología Social con 13 gestores de asistencia indirecta en atención prenatal de alto riesgo, a través de una entrevista presencial grabada en audio y analizada a la luz de la Fenomenología Social. Resultados: el tiempo de espera para acceder al servicio especializado fue de 15 días (32,3\%), de 16 a 30 días $(27,9 \%)$, superior a 90 días $(9,4 \%)$ y $(19,1 \%)$ no tuvo acceso al servicio ambulatorio. Los resultados mostraron como factores que influyen en el tiempo de espera: vacantes insuficientes, información incompleta o sin seguimiento e incumplimiento de los criterios de derivación. Los gestores señalaron que el equipo de salud necesita tener los conocimientos suficientes para desarrollar sus acciones con intenciones recíprocas en el proceso de atención y cuidado. Conclusión: la primera consulta en un servicio especializado se da por un período prolongado y algunas mujeres ni siquiera pueden acceder al servicio, lo que resulta en una atención exclusiva en la atención primaria, que no está organizada para asumir la atención exclusiva de gestantes de alto riesgo.

Palabras clave: Accesibilidad a los servicios de salud; Derivación y consulta; Atención prenatal; Embarazo de alto riesgo; Administración de los servicios de salud; Atención integral de salud.

\section{Introdução}

A gestação é um fenômeno fisiológico e sua evolução, na maioria dos casos, ocorre sem intercorrências, entretanto, algumas gestantes por serem portadoras de alguma doença podem apresentar agravos, com maior probabilidade de evolução desfavorável, colocando em risco a saúde materna ou fetal, fato este que transforma a gravidez em uma gestação de alto risco (AR) (Mirzakhani et al., 2020; Brasil, 2019).

Ao longo dos anos, o Brasil tem investido na qualificação da atenção materno-infantil, observada por meio da instituição do Programa de Humanização no Pré-Natal e Nascimento (PHPN), implantação da Rede Cegonha e da Política Nacional de Atenção Integral à Saúde da Criança (PNAISC), os quais orientam as práticas assistenciais e organizam os serviços de saúde, sendo destaque em políticas públicas por meio da queda da mortalidade materna e infantil no país (Sanine et al., 2019).

Não obstante, ainda persistem alta as taxas de mortalidade materna, sendo esta uma preocupação de saúde mundial (Phommachanh et al., 2019; Balsells et al., 2018). Entre 2000 e 2017 a taxa de mortalidade materna manteve-se inaceitavelmente alta, apresentando uma queda de apenas $38 \%$ no mundo, cerca de 810 mulheres morreram todos os dias, somando 295.000 mortes relacionadas a gravidez e parto em 2017, sendo estas por causas evitáveis (Who, 2019).

A atenção pré-natal (PN) tem como finalidade a redução da morbimortalidade materna e infantil (Tomasi et al., 2017). Entretanto há importantes dificuldades organizacionais (Guimarães et al., 2018). A maioria das mortes maternas poderiam ser evitadas por meio da prevenção ou do gerenciamento de complicações (Who, 2019). Nesse sentido o PN deve envolver ações resolutivas e acolhedoras, por meio da coordenação e do acesso oportuno à rede de Atenção Primária à Saúde (APS), assim como a outros níveis de atenção na rede de serviços de saúde (Guimarães et al., 2018).

Estudos tem avaliado a assistência PN enfocando a cobertura na rede de APS, entretanto pouco se sabe sobre o acesso à assistência pré-natal de alto risco (PNAR) em serviço especializado (SE) (Fernandes et al., 2020). Sendo assim, compreender o seguimento assistencial à gestante de $\mathrm{AR}$, é fundamental para elucidar ações implementadas ou que poderiam ser realizadas 
para o aperfeiçoamento assistencial, assim como, identificar as lacunas destes serviços, favorecendo a implementação de protocolos regionais com ações em políticas públicas de saúde direcionadas à gestação de AR.

Considerando a vulnerabilidade da gestação de AR para maiores agravos maternos e neonatais, o presente estudo tem como objetivo analisar o tempo de espera ao acesso ambulatorial especializado no pré-natal de alto risco.

\section{Metodologia}

Estudo misto com desenho sequencial explanatório, de abordagem quantitativo-qualitativo. Define-se estratégia sequencial explanatória em duas etapas, composta inicialmente por coleta de dados quantitativos, posteriormente análise dos resultados quantitativos e finalmente, planeja-se a segunda fase qualitativa, a partir dos dados quantitativos, follow-up por meio de entrevistas qualitativas, alicerçados na Fenomenologia Social de Alfred Schütz para explicação de respostas confusas, contraditórias ou incomuns (Creswell, 2021).

A etapa quantitativa foi realizada em uma maternidade pública no Sul do Brasil, referência para o parto de AR e a etapa qualitativa foi realizada no ambiente de trabalho dos participantes, sendo estes: Autarquia Municipal de Saúde de Londrina, PR, Brasil; Secretaria Estadual de Saúde da $17^{\text {a }}$ Regional de Saúde, Londrina, PR, Brasil; Ambulatório de Especialidades da Universidade Estadual de Londrina, PR, Brasil e o Consórcio Intermunicipal de Saúde Paranapanema, Londrina, PR, Brasil.

A coleta de dados quantitativa foi realizada no período de outubro de 2016 a agosto de 2017 e a etapa qualitativa foi realizada entre janeiro a março de 2020 por meio de entrevista individual. Na fase quantitativa, realizou-se estudo transversal analítico com puérperas internadas para realização do parto e a etapa qualitativa foi composta por gestores que realizam assistência indireta no PNAR

$\mathrm{Na}$ fase quantitativa os critérios de inclusão foram: mulheres que estivessem no puerpério imediato e internadas na maternidade em estudo, sem comprometimento intelectual e apresentar no mínimo um critério de near miss materno definido pela Organização Mundial de Saúde (Who, 2011). Mulheres que estivessem internadas somente para tratamento clínico foram excluídas do estudo. Para os critérios de inclusão à fase qualitativa, considerou-se profissionais que atuassem no planejamento do acompanhamento à gestante de AR, elegeu-se ainda um tempo mínimo de 60 dias no cargo de gestão.

Para definição do cálculo amostral dos dados quantitativos, considerou-se 1.447 atendimentos realizados no ano de 2015 na instituição pesquisada, com média mensal de 121 mulheres, margem de erro amostral de 5\% e nível de confiança de 95\%, estabelecendo número amostral de 319 mulheres, em concordância com fórmula de (Barbetta, 2012). Na fase qualitativa, utilizou-se amostragem intencional, por meio da identificação dos gestores envolvidos no cuidado indireto à gestante de AR, em serviços de APS e SE ambulatorial no PNAR. Não foi definido a priori o número de participantes, considerou-se os critérios de inclusão à composição da amostra, assegurando-se a participação dos gestores de todas as instituições pesquisadas, sendo as entrevistas encerradas na repetição dos conteúdos originados das falas e alcance do objetivo proposto, obtendo amostra de 13 participantes, identificados pela letra G (Gestor), seguida da numeração de 1 a 13.

A etapa 1 dispôs das seguintes fases: identificação das mulheres com critério de near miss materno, consulta e transcrição dos registros em cartão PN, prontuário hospitalar e entrevista no pós-parto durante a internação hospitalar. Utilizou-se instrumento semiestruturado para o registro dos dados socioeconômicos, demográficos e obstétricos. A coleta de dados da etapa quantitativa foi realizada pela própria pesquisadora com formação stricto sensu, e um grupo de acadêmicos e pós-graduandos lato sensu e stricto sensu, capacitados previamente à coleta de dados. Coletou-se diariamente os dados até a composição da amostra.

A etapa qualitativa teve como referencial teórico metodológico a Fenomenologia Social de Alfred Schütz, por proporcionar a compreensão do significado da gestão PNAR, bem como dos motivos da ação humana que ocorre no mundo da 
vida, frente aos limites assistenciais, quer sejam, por bagagem de conhecimentos insuficiente, ausência de tecnologias leves relacionais no âmbito do cuidar e do cuidado com falhas nas intenções recíprocas para as ações de cuidado e sua resolubilidade, assim como as ações que podem ser implementadas para o aperfeiçoamento assistencial (Schütz, 2012).

A sequência do protocolo da fase qualitativa foi composta por: identificação do gestor envolvido no cuidado indireto à gestante de AR, realizou-se este levantamento por equipe administrativa. Após a identificação do gestor, realizava-se o agendamento da entrevista com a secretária do gestor ou com o próprio gestor, de acordo com a disponibilidade do participante e no próprio local de trabalho do entrevistado. Os relatos foram coletados por entrevista aberta e gravada, cada entrevista teve média de duração de cinquenta minutos, utilizando as seguintes questões norteadoras: Como planeja o cuidado à gestante de AR? Qual estratégia você acredita que poderia ser implementada no acompanhamento PNAR?

A coleta dos dados qualitativos foi realizada pela própria pesquisadora com formação stricto sensu, a qual aprofundou-se do referencial teórico e metodológico adotado.

Os dados quantitativos foram digitados e analisados no software Statistical Package for the Social Sciences (SPSS) versão 20.0, com análise descritiva e analítica por meio do teste Qui-quadrado, para busca de possíveis associações $(\mathrm{p}<0,05)$ entre as variáveis independentes e a dependente "tempo de espera ao acesso à primeira consulta no serviço especializado (SE)”. Após análise quantitativa da assistência PNAR, identificou-se lacunas no acesso ao serviço ambulatorial de referência, emergindo a necessidade de investigação do fenômeno gerenciamento do acesso ao cuidado ambulatorial à gestante de AR, ocorrendo seguimento a etapa qualitativa.

A organização e análise dos dados da fase qualitativa seguiu os princípios teóricos e metodológicos da Fenomenologia Social, sendo estes: leitura acurada de cada relato, obtendo as unidades de sentido da experiência vivida; agrupamento dos aspectos significativos à composição das categorias; análise das categorias, elencando os "motivos porque" que retratam as ações já vivenciadas e expressas e os "motivos para" compondo ações futuras, resultando na categorização dos dados e a compreensão do fenômeno à luz da Fenomenologia Social de Alfred Schütz (Beltrame et al., 2019). Realizou-se correção na transcrição das falas dos depoentes quanto ao português editado à norma culta, não modificando o sentido das falas dos participantes.

Para elucidar a qualidade das informações contidas no instrumento de coleta de dados, elegeu-se a realização de teste piloto com seis puérperas (dados quantitativos) e um gestor (dados qualitativos), sendo realizadas as correções necessárias. Ocorreram conferências manuais e diárias dos questionários preenchidos da fase quantitativa, com a finalidade de conferir questões que não estivessem respondidas, e quando necessário, foi realizado novo contato com a puérpera, no hospital ou por telefone, obtendo as respostas necessárias à pesquisa.

O estudo cumpriu todos os preceitos éticos que regem as pesquisas com seres humanos, sendo que os participantes assinaram o Termo de Consentimento Livre e Esclarecido nas duas etapas da pesquisa. O estudo foi aprovado pelo Comitê de Ética em Pesquisa Envolvendo Seres Humanos da Universidade Estadual de Londrina, sob o no 3.660.461/UEL, em 24 de outubro de 2019 CAAE nº 1597619.5.0000.5231.

\section{Resultados}

$\mathrm{Na}$ fase quantitativa houve predomínio de puérperas que moravam em área urbana $(92,5 \%)$, pouco mais da metade residiam no mesmo município do acompanhamento PN em SE (59,9\%), com faixa etária de idade reprodutiva 20-35 anos $(70,5 \%)$ e extremos da idade (29,5\%). A maior parcela autodeclarou-se branca (57\%), parda ou negra (43\%), com companheiro $(86,8 \%)$ e mais da metade $(57,7 \%)$ das mulheres não apresentavam trabalho com remuneração. Ao analisar os dados obstétricos, $(38,3 \%)$ eram primíparas e $(32,9 \%)$ secundíparas, a maioria $(70,1 \%)$ realizou acompanhamento PN na APS 
concomitantemente com SE, $(18,9 \%)$ teve o acompanhamento PN somente na APS e (11\%) meramente no SE e quanto a via de parto houve predomínio da cesariana $(60,2 \%)$.

Quanto ao tempo de espera para primeira consulta no ambulatório especializado em gestação de alto risco, somente $32,3 \%$ foram encaminhadas até 15 dias e 27,9\% tiveram espera entre 16 a 30 dias. Ressalta-se que dentre as puérperas que tiveram seu parto em maternidade de alto risco $19,1 \%$ não conseguiram acessar o serviço especializado durante seu acompanhamento pré-natal, tendo como justificativas, a ocorrência do parto durante a espera do serviço especializado e o encaminhamento para o alto risco no final da gestação. Salienta-se que 6,3\% das mulheres tiveram acesso ao serviço até dois meses após o encaminhamento, 5,0\% aguardaram até três meses e 9,4\% aguardaram mais que três meses para consulta especializada (Figura 1).

Houve significância estatística entre as características maternas, medida por aspectos socioeconômicos demográficos e histórico obstétrico, com o tempo de espera ao serviço ambulatorial especializado. Dentre as mulheres que tiveram acesso no período de até 30 dias, a grande maioria $(73,0 \%$ - 0,018) estavam na faixa etária à idade reprodutiva favorável, nível educacional de 10 a 12 anos de estudo $(58,8 \%$ - 0,088), residiam em cidade classificada em grande porte $(56,8 \%$ - 0,000), apresentavam patologia prévia à gestação, ainda que sendo pouco mais da metade $(55,7 \%$ - 0,000) e faziam uso de medicamento contínuo $(63,0 \%$ - 0,000) (Tabela 1$)$.

Não houve significância estatística entre a renda familiar com o tempo de espera $(\mathrm{p}=0,142)$, entretanto quase a metade das mulheres que tiveram acesso ao serviço especializado em até 30 dias, possuíam renda familiar superior à três salários mínimos $(48,0 \%)$. Houve diferença estatística entre a realização de 6 ou mais consultas durante o pré-natal e o tempo de espera ao acesso ambulatorial. A maioria das mulheres que tiveram acesso até 30 dias ao serviço especializado, realizaram 6 ou mais consultas durante o seguimento pré-natal $(94,3 \%$ - 0,003) (Tabela 1).

Não houve significância estatística entre o início do acompanhamento pré-natal com o tempo de espera à primeira consulta em ambulatório especializado ( $\mathrm{p}=0,667)$, porém $(22,9 \%)$ das mulheres que não acessaram o serviço especializado, iniciaram o pré-natal tardio, acima de doze semanas de idade gestacional. A realização de ultrassonografia em todos os trimestres gestacionais, não apresentou significância estatística com o tempo de espera $(\mathrm{p}=0,186)$, entretanto $(32,8 \%)$ das mulheres que não tiveram acesso ao serviço especializado, não realizaram no mínimo uma ultrassonografia em todos os trimestres (Tabela 1).

Quanto à idade gestacional na realização do parto, houve significância estatística entre a idade gestacional e o tempo de espera à primeira consulta em serviço especializado. Mulheres que tiveram acesso até 30 dias ao serviço de referência, tiveram idade gestacional a termo acima de 37 semanas de idade gestacional $(76,6 \%$ - 0,002). Ressalta-se que $(42,6 \%)$ das mulheres que não tiveram acesso ao serviço especializado tiveram parto prematuro abaixo de 37 semanas de idade gestacional (Tabela 1). 
Figura 1 - Distribuição do tempo de espera para acesso ao serviço especializado em gestação de alto risco, PR, 2021.

Tempo de espera em dias ao acesso do serviço especializado

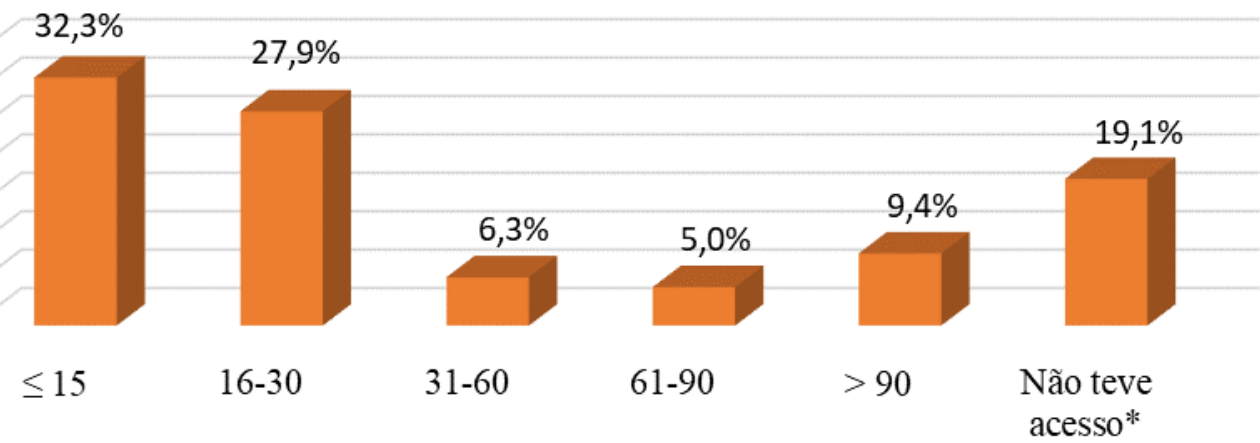

* Não teve acesso ao serviço especializado devido parto ocorrer durante a espera do serviço especializado ou encaminhamento realizado no final da gestação. Fonte: Dados da pesquisa.

Tabela 1 - Distribuição do tempo de espera ao acesso pré-natal em serviço especializado à gestação de alto risco, segundo dados socioeconômicos demográficos, histórico obstétrico, características do cuidado pré-natal e idade gestacional no parto,

PR, 2021.

\begin{tabular}{|c|c|c|c|c|c|c|c|c|c|}
\hline \multirow[t]{3}{*}{ Variáveis } & \multicolumn{9}{|c|}{ Tempo de espera ao acesso a primeira consulta no serviço especializado } \\
\hline & \multicolumn{2}{|c|}{$\leq 30$ dias } & \multicolumn{2}{|c|}{$>30$ dias } & \multicolumn{2}{|c|}{ Não acessou } & \multicolumn{2}{|c|}{ Total } & \multirow[t]{2}{*}{ p valor* } \\
\hline & $\mathrm{n}$ & $\%$ & $\mathrm{n}$ & $\%$ & $\mathrm{n}$ & $\%$ & $\mathrm{n}$ & $\%$ & \\
\hline \multicolumn{10}{|l|}{ Idade em anos } \\
\hline 14-19 & 16 & 8,3 & 5 & 7,6 & 14 & 22,9 & 35 & 11,0 & \multirow[t]{3}{*}{0,018} \\
\hline $20-35$ & 140 & 73,0 & 50 & 75,8 & 35 & 57,4 & 225 & 70,5 & \\
\hline$\geq 36$ & 36 & 18,7 & 11 & 16,6 & 12 & 19,7 & 59 & 18,5 & \\
\hline \multicolumn{10}{|l|}{ Escolaridade } \\
\hline$\leq 9$ anos & 57 & 29,7 & 18 & 27,3 & 16 & 26,2 & 91 & 28,5 & \multirow[t]{3}{*}{0,088} \\
\hline $10-12$ anos & 113 & 58,8 & 31 & 47,0 & 35 & 57,4 & 179 & 56,1 & \\
\hline$>12$ anos & 22 & 11,5 & 17 & 25,7 & 10 & 16,4 & 49 & 15,4 & \\
\hline \multicolumn{10}{|l|}{ Renda familiar** } \\
\hline$\leq 1$ & 35 & 18,2 & 6 & 9,1 & 8 & 13,1 & 49 & 15,4 & \multirow[t]{3}{*}{0,142} \\
\hline $2-3$ & 65 & 33,8 & 22 & 33,3 & 28 & 45,9 & 115 & 36,0 & \\
\hline$>3$ & 92 & 48,0 & 38 & 57,6 & 25 & 41,0 & 155 & 48,6 & \\
\hline \multicolumn{10}{|l|}{ Município**** } \\
\hline Grande porte & 109 & 56,8 & 46 & 69,7 & 36 & 59,0 & 191 & 59,9 & \multirow[t]{3}{*}{0,000} \\
\hline Médio porte & 37 & 19,3 & 7 & 10,6 & 11 & 18,0 & 55 & 17,2 & \\
\hline Pequeno porte & 46 & 23,9 & 13 & 19,7 & 14 & 23,0 & 73 & 22,9 & \\
\hline \multicolumn{10}{|l|}{ Patologia prévia } \\
\hline Sim & 107 & 55,7 & 38 & 57,6 & 16 & 26,2 & 161 & 50,5 & \multirow[t]{2}{*}{0,000} \\
\hline Não & 85 & 44,3 & 28 & 42,4 & 45 & 73,8 & 158 & 49,5 & \\
\hline \multicolumn{10}{|l|}{ Uso de medicação } \\
\hline Sim & 121 & 63,0 & 41 & 62,0 & 13 & 21,3 & 175 & 54,9 & \multirow[t]{2}{*}{0,000} \\
\hline Não & 71 & 37,0 & 25 & 38,0 & 48 & 78,7 & 144 & 45,1 & \\
\hline \multicolumn{10}{|l|}{$\begin{array}{l}\text { Início pré-natal } \leq 12 \\
\text { semanas } \text { IG }^{* * * * *}\end{array}$} \\
\hline Sim & 153 & 79,7 & 55 & 83,3 & 47 & 77,1 & 255 & 79,9 & \multirow[t]{2}{*}{0,667} \\
\hline Não & 39 & 20,3 & 11 & 16,7 & 14 & 22,9 & 64 & 20,1 & \\
\hline \multicolumn{10}{|c|}{$\geq 6$ consultas pré-natal } \\
\hline Sim & 181 & 94,3 & 61 & 92,4 & 49 & 80,3 & 291 & 91,2 & \multirow[t]{2}{*}{0,003} \\
\hline Não & 11 & 5,7 & 5 & 7,6 & 12 & 19,7 & 28 & 8,8 & \\
\hline \multicolumn{10}{|c|}{$\begin{array}{l}\text { Ultrassonografia nos três } \\
\text { trimestres }\end{array}$} \\
\hline Sim & 148 & 77,1 & 53 & 80,3 & 41 & 67,2 & 242 & 75,9 & \multirow{2}{*}{0,186} \\
\hline Não & 44 & 22,9 & 13 & 19,7 & 20 & 32,8 & 77 & 24,1 & \\
\hline Realização do parto & & & & & & & & & \\
\hline$<37$ semanas $\mathrm{IG}^{* * * *}$ & 45 & 23,4 & 11 & 16,7 & 26 & 42,6 & 82 & 25,7 & 0,002 \\
\hline$>37$ semanas $\mathrm{IG}^{* * * * *}$ & 147 & 76,6 & 55 & 83,3 & 35 & 57,4 & 237 & 74,3 & \\
\hline Total & 192 & 100,0 & 66 & 100,0 & 61 & 100,0 & 319 & 100,0 & \\
\hline
\end{tabular}

*Teste Qui-quadrado (p<0,05). **Base do salário mínimo do ano 2017 - R \$980,00. ***Porte do município residencial da gestante de acordo com Instituto Bra Geografia e Estatística (IBGE, 2021). ****Idade gestacional. Fonte: Dados da pesquisa. 
$\mathrm{Na}$ fase do estudo qualitativo a idade dos gestores variou de 36 a 62 anos, houve predomínio de servidor público (77\%) e do sexo feminino $(84,6 \%)$. A maioria eram enfermeiros $(69,2 \%)$ e os demais profissionais da medicina, farmácia e línguas estrangeiras $(30,8 \%)$. O tempo de formação teve média de vinte e dois anos. Foi unânime os participantes possuírem pós-graduação, com lato sensu $(69,2 \%)$ e stricto sensu $(30,8 \%)$.

Da análise dos relatos, desvelou-se um contexto de significados vivenciados até o momento "motivos por que", apresentadas nas três primeiras categorias: Gerenciamento do acesso ao SE; Interferência no tempo de espera e Limites da gestão e gerenciamento do cuidado. As expectativas descritas nos "motivos para", resultou na categoria: Possibilidades no gerenciamento do PNAR.

\section{Gerenciamento do acesso ao SE}

$\mathrm{O}$ tempo prolongado para acesso à primeira consulta no $\mathrm{SE}$, desvelou-se como uma lacuna no acompanhamento PNAR, evidenciada por meio de uma longa lista de espera, com impacto no processo do gerenciamento, de uma demanda represada que durante o tempo de espera, é acompanhada somente pela APS a qual não está organizada para atender de maneira eficaz as necessidades da gestante de AR de forma recíproca.

Temos uma lista expectante que são das gestantes que vem, e nós não damos conta de atender todas, algumas vão ter que esperar de três a quatro meses, para iniciar o atendimento. (G5)

Às vezes essa gestante chega tardiamente na referência, e então a UBS fica com ela, o que não deveria, onde o que deveria ser parceria fica segurando essa bomba. (G12)

\section{Interferência no tempo de espera}

Esta categoria revela as causas para o tempo de espera prolongado ao SE, como, oferta insuficiente, aumento de mulheres com gestação de AR, uso indiscriminado de medicamentos controlados, dados insuficientes no encaminhamento, não atendimento aos critérios de encaminhamento para o AR e não monitoramento das gestantes referenciadas.

Temos dois prestadores para alto risco que em determinados momentos do ano temos redução da oferta de vagas.

Temos observado uma quantidade muito grande de gestantes em qualquer idade com comorbidades ou gestantes utilizando medicamentos sem muito critério. $O$ abuso de medicamento na saúde mental é muito grande, é assustador em qualquer idade. (G3)

Tudo depende se o encaminhamento for bem feito, com os dados necessários e que atenda aos critérios. Se faltou informações devolvemos como dados insuficientes. Também temos caso que na consulta o médico identifica que não tinha a necessidade do encaminhamento, com encaminhamentos realizados de forma incorreta. (G4)

O encaminhamento com dados insuficientes, e até o não monitoramento das gestantes referenciadas (G12). 


\section{Limites da gestão e gerenciamento do cuidado}

Dentre os limites para o aumento do número de vagas revelou-se a escassez financeira para ampliação do espaço físico e recursos humanos suficientes nas especialidades necessárias. A não continuidade das ações planejadas foi identificada como impedimento à continuidade das ações, decorrente das emergências epidêmicas e mudanças da gestão municipal.

Nós crescemos bastante nos últimos anos, e desenvolvemos vários tipos de atendimentos diferenciados em rede e o espaço físico ficou pequeno. (G4)

O nosso grande desafio é ter profissionais nas especialidades necessárias e em quantidades suficientes para atender as gestantes. Não temos recurso próprio, ou é recurso do município, ou do Estado, ou do federal, então é por meio deste recurso que recebemos que vamos conseguindo contratar profissionais para fazer esse atendimento.

Tem outras coisas que te atropelam, a epidemia de dengue, toda regional se volta para isso, chega o coronavírus e você não consegue dar continuidade àquilo que você planejou. Esse é o último ano da gestão municipal, então quando muda a gestão municipal, muda todas as pessoas que estão à frente e começa todo o processo de novo. (G6)

A implantação de políticas públicas foi identificada com lacunas entre as ações determinadas em nível federal e as necessidades regionais, prejudicando o planejamento local. Os cargos ocupados por gestores municipais, com foco político e sem formação na área de saúde, foram identificados como dificultadores para o entendimento das necessidades nos serviços de saúde.

Nós temos um processo de implantação de políticas verticalizado, com iniciativa do governo do Estado, então os municípios se apresentam como polo passivo na implantação de políticas públicas. Temos dificuldade de formular um plano regional. (G7)

Temos secretários de saúde que não tem nenhuma base de saúde, estas pessoas não têm formação na área da saúde, eles são políticos. Então não conseguimos explicar a ele que um município de 4.000 habitantes, que tem uma UBS e um enfermeiro, é impossível implantar alguma coisa ou solicitar a presença do enfermeiro em todas as reuniões e nos treinamentos. (G9)

\section{Possibilidades no gerenciamento do PNAR}

Os participantes trouxeram suas expectativas quanto ao aperfeiçoamento da assistência PNAR, com enfoque na necessidade do cuidado integral e interdisciplinar. O aumento da oferta de vagas no SE foi marcante, tendo como estratégia a esta necessidade o investimento financeiro na ampliação da área física dos ambulatórios, aumento de recursos humanos qualificados e manutenção de materiais.

As melhorias no atendimento das unidades de APS, foram desveladas como dependentes de aprimoramento dos critérios de encaminhamento, assim como da expansão de capacitação a equipe da APS, por profissionais do SE, almejando aquisição de bagagem de conhecimento suficiente, para o desenvolvimento das ações no cuidado. 
Mais completo possivel, acolhedor, com assistência de diversos profissionais, desde o agente comunitário até o administrativo. Precisamos expandir nossas ações de capacitação, por exemplo o serviço de alto risco poderia ter uma parceria conosco, onde a rede de referência poderia capacitar os nossos profissionais da atenção primária, com algumas condições que fossem pertinentes a atenção primária. (G2)

Precisamos de recurso financeiro para ampliar a área física e contratar mais profissionais. O recurso financeiro também é importante, para manter os materiais. (G4)

Teria que ter melhorias do atendimento na atenção básica, para que viessem para o serviço especializado só os casos mais necessários. (G5)

Desvelou-se também a necessidade da carreira na gestão de serviços, com ênfase e formação na área da saúde, livre de influências políticas, para implementação das políticas públicas.

Deveríamos ter mais pessoas nos cargos de uma maneira geral, que tivessem uma formação mínima na área e que tivesse uma visão de fato, eu estou dizendo especificamente da saúde, do que é saúde pública, eu acho que aquilo que está dando certo deve se tornar uma política de Estado e não uma política de governo. (G6)

\section{Discussão}

O estudo misto ensejou a análise do acesso ao acompanhamento especializado no PNAR, por meio do tempo de espera aguardado pela gestante, assim como das características e cuidados que podem interferir no período aguardado, ademais do gerenciamento da gestão frente aos desafios da lista de espera e possibilidades que podem ser utilizadas para minimizar as lacunas encontradas. No presente estudo, evidenciou-se o não acesso imediato ao SE, sendo que a primeira consulta ambulatorial não ocorreu nas primeiras semanas após o encaminhamento, assim como, uma parcela de gestantes não foi contrarreferenciada, evidenciando a não integração do cuidado entre os serviços da APS e SE, não havendo reciprocidade de intenções entre quem requer o cuidado e quem cuida.

Neste sentido, a fragmentação da assistência é apontada na literatura como um dos maiores obstáculos na rede de APS para a continuidade assistencial (Soares \& Higarashi, 2019).

$\mathrm{O}$ acesso ao SE em até 30 dias, foi influenciado pela idade materna, nível educacional, porte do município residencial da gestante, possuir patologia prévia à gestação, uso de medicamento contínuo, número de consultas durante o PN, e IG no nascimento. Um estudo internacional identificou que mulheres mais jovens, assim como baixa escolaridade, são fatores que influenciam no acesso tardio ou não acesso da assistência (Blakeney et al., 2019).

A vulnerabilidade da gestante de $\mathrm{AR}$, suscita a necessidade de acompanhamento com maior desvelo, sendo necessário o acompanhamento PN da APS, concomitante do SE, em tempo oportuno à prevenção de agravos. Ademais do acesso aos serviços, o PNAR deve ter enfoque ao cuidado integral e interprofissional, segundo a especificidade de cada gestante, proporcionando a continuidade do cuidado gestacional, além da segurança e apoio às equipes.

Apesar do princípio doutrinário da universalidade do Sistema Único de Saúde (SUS), a gestante de AR vivencia dificuldades no acesso à rede de atenção, principalmente no SE, deparando-se com fila de espera, independente da sua condição e IG (Brilhante \& Jorge, 2020; Monteiro et al., 2019).

Quanto à via de parto, houve predomínio da cesárea, corroborando com a literatura nacional e internacional (Monteiro el al., 2019; Leal et al., 2017; Schantz et al., 2016). O parto cirúrgico vem crescendo significativamente, sendo motivo de 
preocupação, considerado pela Organização Mundial da Saúde uma epidemia de cirurgia cesárea, com alta incidência em países desenvolvidos e subdesenvolvidos (Monteiro et al., 2019; Fernandes et al., 2020). A indicação da cesárea deve ser realizada com prudência, sendo este um dos grandes desafios relacionados à redução da mortalidade materna e neonatal, entretanto, quando indicada de maneira indiscriminada associa-se a maior risco de desfecho materno grave (Mascarello, Horta \& Silveira, 2017).

O acesso ao SE durante o PNAR, surge no mundo da vida, por meio das experiências vivenciadas (motivos porque), e evidenciadas por não suprimento da lista de espera e número de oferta de vagas no SE. O típico da ação na gestão PNAR, desvelou-se no contexto de significados no gerenciamento do SE. No presente estudo, desvelaram-se inúmeros fatores que podem influenciar no tempo de espera para o SE, desde a incompatibilidade de oferta versus aumento da demanda, assim como a incompletude dos dados necessários ao encaminhamento, não atendimento aos critérios que classificam a gestação de AR e não monitoramento das gestantes referenciadas.

Salienta-se que o subfinanciamento de recurso federal, leva a limitação dos serviços especializados, resultando em incompatibilidade entre a oferta e demanda, ademais, a demanda reprimida por procedimentos de média e alta complexidade impacta na restrição do acesso, inviabilizando o atendimento às necessidades de saúde, comprometendo a integralidade do cuidado (Monteiro et al., 2019). Frente ao gerenciamento do tempo de espera para o SE, as necessidades epidêmicas, assim como as mudanças da gestão municipal, foram identificadas como impedimento à continuidade das ações planejadas.

O processo de implementação de políticas públicas em saúde, deve partir de um pressuposto teórico, passando por um ciclo de seis estágios: definição do problema, realização do diagnóstico causal, desenvolvimento de planos e então, deve ser tomada uma decisão política sobre as reformas iniciais, sua implementação e avaliação do seu impacto. Entretanto, esta sequência lógica e linear raramente acontece na realidade, tendo seu início em diferentes locais, não seguindo todas as etapas deste processo, ou fazendo com que todos os estágios aconteçam de uma única vez (Campos \& Reich, 2019).

A necessidade de implantação de políticas públicas que atendam à necessidade regional, foi identificada como uma particularidade do município estudado, haja visto que tal município é referência para várias regiões e outras especialidades. Neste sentido, desvelou-se a importância de gestores e promotores da saúde possuírem planos gerenciais com foco nas necessidades reais da saúde pública, não permitindo que interesses políticos mudem o direcionamento de tais políticas.

Ressalta-se os avanços alcançados ao longo dos anos na assistência PN, por meio do acesso PN universal na maioria dos estados brasileiros, entretanto, persistem fragilidades assistenciais, quanto ao acesso a realização dos exames, em especial as ultrassonografias (Sanine et al., 2019; Brilhante \& Jorge, 2020).

No presente estudo, houve predomínio de gestantes com início precoce do PN, sendo este um valioso recurso à identificação e encaminhamento da gestante com maior risco. Considerando a parcela de mulheres que iniciaram seu PN após 12 semanas de IG, e que não conseguiram o atendimento em SE à gestação de AR, ressalta-se a importância da primeira consulta ocorrer precocemente na APS, favorecendo o encaminhamento ao SE, se necessário, o mais breve possível.

Foi marcante a realização de ultrassonografia em todos os trimestres gestacionais, entretanto uma parcela das gestantes que não tiveram acesso ao SE, não tiveram ultrassom trimestral realizado. Salienta-se que no PNAR, pode ser fundamental a realização de ultrassonografias e exames específicos, sendo que a APS, muitas vezes não tem acesso em momento oportuno a tais exames peculiares.

Diante das dificuldades ao acesso PN em SE, doravante a bagagem de conhecimento dos gestores participantes, desvelaram-se expectativas às ações futuras (motivos para) ao acesso do serviço ambulatorial (Schütz, 2012). Os participantes relataram a necessidade do cuidado integral e interdisciplinar, da relação face a face e reciprocidade de intenções entre quem cuida e quem requer o cuidado, bem como, disposição de recurso financeiro à obtenção do aumento de vagas e área física, além da manutenção de materiais. $\mathrm{O}$ acesso à rede de atenção à saúde apresenta uma grande magnitude social, pouco 
compreendida e reconhecida por gestantes de AR e profissionais, sendo necessário ações efetivas à integração das redes de APS e SE; garantia de acesso ao PN de qualidade, medicamentos e exames em tempo oportuno; regionalização da atenção especializada; fortalecimento da referência e contra referência, assim como sua inclusão nos processos de formação profissional e educação em saúde à comunidade (Brilhante \& Jorge, 2020).

No presente estudo, os gestores sugerem como estratégia ao acesso de vagas no SE, a atuação do serviço de referência, por tempo determinado à estabilização clínica da gestante, com continuidade ao tratamento prescrito no SE por meio da APS, ocorrendo nova consulta ambulatorial caso a UBS identifique a necessidade. Ressalta-se que para atuação do SE como interconsulta, com objetivo de estabilização clínica, o tempo de espera ao SE deve ser efetivo, com menor tempo possível para o acesso, estabelecimento de referência e contra referência efetiva, número de profissionais suficientes na APS, além do contato facilitado entre equipe de APS e SE. Neste contexto, não necessariamente uma gestante acompanhada no SE, deve manter-se em consultas subsequentes no ambulatório de risco, entretanto os serviços devem estar organizados para essa implementação do protocolo.

Outro fator importante para melhorias no acesso ao SE, desvelou-se por meio do aprimoramento dos critérios de encaminhamento, assim como expansão das capacitações, alcançando maior número de pessoas habilitadas, considerou-se os profissionais do SE como instrutores à capacitação. O treinamento de curta duração resulta em maior bagagem de conhecimento e habilidades dos profissionais de saúde, além da melhoria da prática clínica. A oportunidade para o aperfeiçoamento profissional, promove retenção do conhecimento e habilidade a longo prazo, obtidos após o treinamento, com impacto em melhor resultado da saúde materna e perinatal (Ameh et al., 2019).

Apesar dos avanços alcançados na assistência PN, assim como o protocolo instituído no município estudado, o seguimento PNAR apresenta desafios peculiares às necessidades regionais, de suporte e fragilidades ao encaminhamento da gestação de AR. Neste sentido, salienta-se a necessidade dos cargos ocupados por gestores e promotores públicos em saúde, possuírem formação em saúde pública, direcionando a tomada de decisão, manutenção e implementação de políticas bemsucedidas. Sugere-se ainda, que gestores municipais e estaduais, possam participar ativamente na implantação e implementação de políticas públicas, com participação e decisões junto a entes federativos.

Aponta-se como limitação deste estudo a amostra específica em uma única região, o qual não foi possível sua generalização, entretanto os resultados são representativos e podem servir como base à novos estudos. As lacunas identificadas no acesso do SE, assim como, as possibilidades apontadas ao gerenciamento assistencial, pode direcionar gestores públicos, à implantação e implementação de políticas públicas na assistência gestacional de AR, por meio da identificação das falhas e ações assistenciais que podem ser desenvolvidas ao aprimoramento assistencial, repercutindo na promoção e prevenção dos agravos materno e neonatal.

\section{Considerações Finais}

Conclui-se que o acesso à primeira consulta em SE à gestação de AR, ocorre em tempo prolongado, assim como, algumas mulheres não conseguem nem mesmo o acesso ao serviço durante seu PN, carecendo de relação face a face, o que permitiria bagagem de conhecimento para vivenciar com qualidade todo o ciclo gravídico e puerperal, resultando no cuidado exclusivo por meio da APS, a qual não está organizada para assumir esse cuidado à gestante de AR, comprometendo a intenção recíproca de cuidar e de ser cuidada. Identificou-se que o acesso ao SE em até 30 dias, foi influenciado por idade materna, nível educacional, porte do município residencial da gestante, possuir patologia prévia, uso de medicamento contínuo, número de consulta PN e IG no nascimento.

Quanto às estratégias a serem implementadas, sugere-se o acesso ao SE como interconsulta, o aprimoramento dos critérios de encaminhamento de risco gestacional e expansão das ações de capacitação aos profissionais da APS. 
Destaca-se a importância da participação ativa de gestores municipais e estaduais na implantação e implementação de políticas efetivas, ademais da necessidade dos cargos ocupados por tomadores de decisão, apresentarem formação em saúde pública, livre de interesses políticos, almejando melhorias assistenciais no acesso do SE.

Sugere-se novos estudos sobre a temática em questão, tendo como amostra gestores do SUS e de outros níveis federativos, por meio da investigação fenomenológica.

\section{Referências}

Ameh, C. A., Mdegela, M., White, S., \& Broek, N. V. D. (2019). The effectiveness of training in emergency obstetric care: a systematic literature review. Health Policy and Planning, 34(4), 257-270. https:/www.researchgate.net/publication/333291792_The_effectiveness_of_training_in_emergency_obstetric_ care_A_systematic_literature_review. https://doi.org/10.1093/heapol/czz028.

Balsells, M. M. D., Oliveira, T. M. F., Bernardo, E. B. R., Aquino, P. S., Damasceno, A. K. C., Castro, R. C. M. B., Lessa, P. R. A., \& Pinheiro, A. K. B. (2018). Evaluation of prenatal care process for habitual-risk pregnant women. Acta Paul Enferm, 31(3), 247-254. https://www.scielo.br/j/ape/a/kvhNQDDLRvTMdb5Tr4cKsJr/?format=pdf\&lang=en. http://dx.doi.org/10.1590/1982-0194201800036.

Barbetta, P. A. (2012). Estatística aplicada às ciências sociais, UFSC.

Beltrame, C. H., Batista, F. F. A., Caldeira, S., Zani, A. V., Cardelli, A. A. M., \& Ferrari, R. A. P. (2019). Mothers' experience in following up their newborns: a phenomenological study. Online braz. j. nurs., 18(2). http://www.objnursing.uff.br/index.php/nursing/article/view/6270. https://doi.org/10.17665/16764285.20196270.

Blakeney, E. L., Herting, J. R., Bekemeier, B., \& Zierler, B. K. (2019). Social determinants of health and disparities in prenatal care utilization during the Great Recession period 2005-2010. BMC Pregnancy Childbirth, 19(390). https://www.ncbi.nlm.nih.gov/pmc/articles/PMC6819461/pdf/12884_2019_Article_ 2486.pdf. https://doi.org/10.1186/s12884-019-2486-1.

Brasil, Ministério da Saúde. (2019). Nota técnica para organização da rede de atenção à saúde com foco na atenção primária à saúde e na atenção ambulatorial especializada. Saúde da mulher na gestação, parto e puerpério. Guia para as secretarias estaduais e municipais de saúde. Brasília: Ministério da Saúde.

Brilhante, A. P. C. R., \& Jorge, M. S. B. (2020). Institutional violence in high-risk pregnancy in the light of pregnant women and nurses. Rev. Bras. Enferm., 73(5). https://www.scielo.br/j/reben/a/p8gY73vcLRChsq84ZvVTgjL/?format=pdf\&lang=en. http://dx.doi.org/10.1590/0034-7167-2018-0816.

Campos, P. A., \& Reich, M. R. (2019). Political Analysis for Health Policy Implementation. Health Systems \& Reform., 5(3), 224-235. https://www.tandfonline.com/doi/epub/10.1080/23288604.2019.1625251 ?needAccess=true. https://doi.org/10.1080/23288604.2019.1625251.

Creswell, J. W. (2021). Projeto de pesquisa: métodos qualitativo, quantitativo e misto, Penso.

Fernandes, J. A., Venâncio, S. I., Pasche, D. F., Silva, F. L. G., Aratani, N., Tanaka, O. Y., Sanine, P. R., \& Campos, G. S. (2020). Assessment of care for high-risk pregnancy in four Brazilian metropolises. Cad. Saúde Pública, 36(5). https://www.scielo.br/j/csp/a/TpXB8XV3DMg6YcWrGwK4gQm/?format=pdf \&lang=pt. https:// 10.1590/0102-311X00120519.

Guimarães, W. S. G., Parente, R. C. P., Guimarães, T. L. F., \& Garnelo, L. (2018). Access to prenatal care and quality of care in the Family Health Strategy: infrastructure, care, and management. Cad Saúde Pública, 34(5). https://www.scielo.br/j/csp/a/9CMWjGgNGcLLYRjpCQQrymh/?lang=pt. https://doi.org/10.1590/0102-311X00110417.

Leal, R. C., Santos, C. N. C., Lima, M. J. V., Moura, A. O. P., Pedrosa, A. O., \& Costa, A. C. M. (2017). Maternal-perinatal complications in high risk pregnancy. Journal of nursing UFPE On Line, 11(4), 1641-1649. https://pesquisa.bvsalud.org/portal/resource/pt/bde-31304. https://doi.org/10.5205/reuol.10438-93070-1.

Mascarello, K. C., Horta, B. L., \& Silveira, M. F. (2017). Maternal complications and cesarean section without indication: systematic review and metaanalysis. Rev Saude Publica, 51(105). http://www.rsp.fsp.usp.br/artigo/complicacoes-maternas-e-cesarea-sem-indicacao-revisao-sistematica-e-meta-analise. https://doi.org/10.11606/S1518-8787.2017051000389.

Mirzakhani, K., Ebadi, A., Faridhosseini, F., \& Khadivzadeh, T. (2020). Well-being in high-risk pregnancy: an integrative review. BMC Pregnancy Childbirth, 20(1). https://bmcpregnancychildbirth.biomedcentral.com/track/pdf/10.1186/s12884-020-03190-6.pdf. https://doi.org/10.1186 /s12884-020-031906.

Monteiro, M. F. V., Barbosa, C. P., Vertamatti, M. A. F., Tavares, M. N. A., Carvalho, A. C. O., \& Alencar, A. P. A. (2019). Access to public health services and integral care for women during the puerperal gravid period period in Ceará, Brazil. BMC Health Serv Res., 19(851), 1-8. https://www.readcube.com/articles/10.1186\%2Fs12913-019-4566-3. https://doi.org/10.1186/s12913-019-4566-3.

Phommachanh, S., Essink, D. R., Jansen, M., Broerse, J. E. W., Wright, P., \& Mayxay, M. (2019). Improvement of Quality of Antenatal Care (ANC) Service Provision at the Public Health Facilities in Lao PDR: Perspective and Experiences of Supply and Demand Sides. BMC Pregnancy Childbirth, 19(255). https://www.researchgate.net/publication/334621024_Improvement_of_Quality_of_Antenatal_Care_ANC_Service_Provision_at_the_Public_Health_Facilitie s_in_Lao_PDR_Perspective_and_Experiences_of_Supply_and_Demand_Sides. https://doi.org/10.1186/s12884-019-2345-0.

Sanine, P. R., Venancio, S. I., Silva, F. L. G., Aratani, N. M. M. L. G., \& Tanaka, O. Y. (2019). Prenatal care in high-risk pregnancies and associated factors in the city of São Paulo, Brazil. Cad. Saúde Pública, 35(10). https://www.scielo.br/j/csp/a/SVF7DzHMnbYKd36j8kBmZ7k/?lang=pt. https://doi.org/10.15 90/0102-311X00103118. 
Research, Society and Development, v. 11, n. 1, e16611124626, 2022

(CC BY 4.0) | ISSN 2525-3409 | DOI: http://dx.doi.org/10.33448/rsd-v11i1.24626

Soares, L. G., \& Higarashi, I. H. (2019). Case management as a high-risk prenatal care strategy. Rev. Bras. Enferm., 72(3), 692-699. https://www.scielo.br/j/reben/a/JpqvH67cTJX3HyknTkkzNDy/abstract/?lang=en. http://dx.doi.org/10.1590/0034-7167-2018-0483.

Schantz, C., Sim, K. L., Petit, V., Rany, H., \& Goyet, S. (2016). Factors associated with caesarean sections in Phnom Penh, Cambodia. Reprod Health Matters, 24(48), 111-121. https://www.tandfonline.com/doi/pdf/10.1016/j.rhm.2016.11.009? needAccess=true. https://dx.doi.org/10.1016/j.rhm.2016.11.009.

Schütz, A. (2012). Sobre fenomenologia e relações sociais, Vozes.

Tomasi, E., Fernandes, P. A. A., Fischer, T., Siqueira, F. C. V., Silveira, D. S., Thumé, E., Duro, S. M., Saes, M. O., Nunes, B. P., Fassa, A. G., \& Facchini, L. A. (2017). Quality of prenatal services in primary healthcare in Brazil: indicators and social inequalities. Cad. Saúde Pública, 33(3). https://europepmc.org/article/med/28380149. http://doi.org/10.1590/0102-311X00195815.

World Health Organization. (2019). Maternal mortality. WHO. https://www.who.int/en/news-room/fact-sheets/detail/maternal-mortality.

World Health Organization (2011). Evaluating the quality of care for severe pregnancy complications: the WHO near-miss approach for maternal health. WHO. https://apps.who.int/iris/bitstream/handle/10665/44692/9789241502221_eng.pdf?sequence=1. 